\title{
Resistencia de Bacterias Enteropatógenas a Antimicrobianos de Uso Habitual
}

\author{
Dra. Valeria Prado J.1; T.M. Lucía Reyes M.1; T.M. Maria Rosalba Urbina C. ${ }^{2}$; \\ B.Q. María OJga Phillips F. 2
}

Resistance of Enteropathogen Bacteria to Commonly Used Antibiotics

In vitro susceptibility of 368 enteropathogenic bacterial strains isolated from acute intestinal infections were studied, including 100 strains of Enteropathogenic E. Coli (E.P.E.C.), 85 Shigella specimens, 100 isolates of Salmonella typhimurium and 83 of Salmonella typhi. Minimum irhibitory concentrations (MIC) were determined for Ampicilin, Chloramphenicol, Cotrimoxazole, Gentamicin, Dibekacin, Amikacin, Cefotaxim and Ceftriaxone. Neomicin and Polymyxin $B$ were also tested against EPEC strains. The resistance pattern of each strain was analyzed. Only 46\% of EPEC were sensitive to Neomicin whereas Polymyxin B was active againts $100 \%$ of them. 75 to $85 \%$ of Shigellae were sensitive to Ampicillin, Cotrimoxazole and Chloramphenicol. Salmonella typhimurium strains were resistant to one or more of the studied antibiotics in $50 \%$ of the cases. Among the tesistant strains prevailed a common pattexn of multiple-resistance to five antibiotics (Ampicilin, Gentamicin, Dibekacin, Chloramphenicol, Cotrimoxazole). Amikacin, Cefotaxime and Ceftriaxone were active against all tested Salmonella typhimurium isolates. All Salmonella typhi strains $(100 \%$ were sensitive to the eight tested antibiotics. Clotamphenicol and Cotrinoxazole demonstrated good levels of activity, thus remaining the drugs of choice in typhoid fever thcrapy in our setting.

Las infecciones entéricas bacterianas son causa frecuente de morbilidad en nuestro país. Dentro de ellas tenemos que considerar los casos de diarrea aguda que constituyen entre el 18 a $20 \%$ de las consultas en los niños menores de 3 años y la primera causa de hospitalización en los meses de primavera-verano en el grupo de lactantes menores de 1 año, de estrato socio-económico bajo que se atienden en los servicios estatales de salud ${ }^{1}$.

En estudios de etiología de diarrea aguda desarrollados anteriormente por nuestro grupo, hemos podido constatar que en más te la mitad de los casos se aislan agentes bacterianos ${ }^{2-3}$.

Por otra parte la fiebre tifoídea en los últimos cinco aflos presenta una tendencia ascendente en nuestro país y la incidencia de la enfermedad ha superado los 120 casos por 100.000 habitantes 4 .

Frente a la diarrea aguda el enfoque terapéutico está dirigido fundamentalmente a la corrección de la deshidratación y de los trastornos del equilibrio ácido-base, sin embargo el uso de antimicrobianos ha demostrado ser eficaz en el control de los síntomas causados por determina-

1 Unidad Microbiología, Depto. Salud Pública. Div. Cs. Med. Oriente, Facultad de Medicina, Unversidad de Chile.

2 Alumnas Programa Magister en Ciencias, Mención Microbiología, Facultad de Medicina, Universidad de Chile. dos gérmenes y de la aparición de casos secunda. rios, al erradicar precozmente el agente infeccioso. De esta manera se obtienen beneficios en pacientes con diarrea por $\mathbf{E}$. coli clásicos (ECEP) mediante tratamiento con antibióticos no absorbibles (de acción local) y en la Shigellosis está indicado el uso de antibióticos de buena absorción que alcancen concentraciones piasmáticas adecuadas, atendiendo a los diferentes mecanismos de patogenicidad de ambos agentes s-6-7.8. $^{\text {. }}$ En cambio en las infecciones enterales provocadas por Salmonellas zoonóticas entre ellas $S$. typhimurium ningún antimicrobiano tiene utilidad y está contraindicado excepto cuando producen infecciones sistémicas. El tratamiento antimicrobiano en la fiebre tifoidea ha mejorado significativamente el pronóstico de esta enfermedad y el Cloranifenicol sigue siendo la droga de elección, sin embargo es necesario mantener bajo vigilancia la sensibilidad de las cepas de $\mathrm{S}$. typhi pues en otros países se han observado cepas con resistencia de otigen plasmidjal a varios antibióticos, que han causado serios brotes epidémicos como el de México en 19729:10-11 .

Considerando los antecedentes expuestos intentamos delinear el patrón de sensibilidad actual de las bacterias anteropatógenas más frecuentes en nuestro medio a los antimicrobianos de uso habitual, para dar una orientación racional al tratamiento antimicrobiano y vigilar la eventual aparición de resistencia en este grupo bacterjano. 


\section{MATERIAL Y METODOS}

$\mathrm{Se}$ incluyeron en el estudio 368 cepas de bacterias enteropatógenas, algunas aisladas de lactantes con diarrea aguda del Area Metropolitana Oriente de Santiago y otras de pacientes con tifoídea. Una parte de las cepas fue suministrada por la Dra. Karen D'Ottone, Jefe del Centro de Referencia de enterobacterias del Instituto de Salud Pública. Todas ellas fueron aisladas en un período de 2 años que terminó en Marzo de 1984.

Se estudiaron 100 cepas de ECEP (34 serogrupo 0111,20 serogrupo 0119,17 serogrupo 055 , 6 serogrupo 018,5 serogrupo 086,5 serogrupo 020, 4 serogrupo 026, 3 serogrupo 0125,2 serogrupo 0126,2 serogrupo 0127,1 cepa serogrupo 044 y una 0142) 85 de Shigella (46 Sh. flexnerii, $29 \mathrm{Sh}$. sonnei, $10 \mathrm{Sh}$. boydii) $100 \mathrm{de}$ Salmonella enteritidis serotipo typhimurium y 83 de Salmonella typhi.

La sensibilidad in vitro se evaluó midiendo la concentración inhibitoria minima (CIM) en $\mathrm{mcg} / \mathrm{ml}$ por técnica de dilución en placa con Ampicilina, Cloramfenicol, Cotrimoxazol, Gentamicina, Dibekacina, Amikacina, Cefotaxime y Ceftriaxone. Las cepas de ECEP se estudiaron además con Neomicina y Polymixina B. Se emplearon diluciones seriadas en un rango entre $0,25 \mathrm{mcg} / \mathrm{ml}$ y $256 \mathrm{mcg} / \mathrm{ml}$ de cada antimicrobia. no. Como medio de cultivo se usó agar Müller Hinton y agar DST con $5 \%$ de sangre humana para el estudio de Cotrimoxazol. El inóculo de cada cepa se ajustó a una densidad bacteriana de $10^{5} \mathrm{UFC} / \mathrm{ml}$ (unidades formadores de colonias $/ \mathrm{ml}$ ) a partir de un cultivo en caldo de 18 hrs, y se sembró $0,002 \mathrm{ml}$ mediante el múltiple inoculador de Steers.

Para comparar la actividad de los diferentes antimicrobianos se utilizó como parámetro la CIM 50 y la CIM 90 (CIM que era capaz de jnhibir el $50 \%$ y $90 \%$ de las cepas respectivamen. te). Se identificaron además las cepas sensibles, considerando para este criterio como CIM útil los siguientes valores en $\mathrm{mcg} / \mathrm{ml}$ de acuerdo a pautas internacionales ${ }^{13}$ : Ampicilina 16, Cloramfenicol 12,5, Gentamicina 4, Dibekacina 4, Amikacina 16, Neomicina 16, Cotrimoxazol 80, Polimixina B 8, Cefotaxime 16 y Ceftriaxone 16.

\section{RESULTADOS}

La sensibilidad de 100 cepas de ECEP a 10 antibióticos se muestra en la Tabla 1. La Neomicina demostró escasa actividad, con CIM 50 y 90 muy elevadas y un número reducido de cepas sensibles (46\%); La Polimixina fue activa para todas las cepas estudiadas con CIM 50 y CIM 90 ,
16 y 32 veces más bajos que la Neomicina respectivamente.

Tabla 1.

Actividad comparativa in vitro (CIM $\mathrm{mcg} / \mathrm{ml}$ ) de 100 cepas de ECEP frente a 10 antimicrobianos

$\begin{array}{llr}\text { CIM 50 } & \text { CIM 90 } & \text { \% cepas } \\ \mathrm{mcg} / \mathrm{ml} & \mathrm{mcg} / \mathrm{ml} & \text { sensibles }\end{array}$

$\begin{array}{lrrr}\text { Neomicina } & 64 & \geqslant 256 & 46 \\ \text { Polimixina B } & 4 & 8 & 100 \\ \text { Ampicilina } & \geqslant 256 & \geqslant 256 & 18 \\ \text { Cloramfenicol } & 4 & \geqslant 256 & 67 \\ \text { Gentanlicisa } & 0,5 & 1 & 99 \\ \text { Dibekacina } & 1 & 1 & 99 \\ \text { Amikacina } & 2 & 2 & 100 \\ \text { Cotrimoxazole } & 4 & 256 & 58 \\ \text { Cefotaxime } & \$ 0.25 & \leqslant 0.25 & 100 \\ \text { Ceftriaxone } & \leqslant 0.25 & \leqslant 0.25 & 100\end{array}$

$\mathrm{ECEP}=\mathrm{E}$, coli enteropatógenos clásicos.

En los restantes antibióticos estudiados, que se incluyeron como marcadores de referencia, se observó muy pobre actividad de Ampicilina con sólo $18 \%$ de cepas sensibles y los más altos niveles de ClM. Cotrimoxazol y Cloramfenicol tuvieron actividad intermedia. Los aminoglicósidos mostraron buena actividad con CIM entre 0,5 y $2 \mathrm{mcg} / \mathrm{ml}$ y casi total sensibilidad de las cepas; las cefalosporinas de tercera generación resultaron con las mayores actividades in vitro.

En la Tabla 2 se expone la actividad in vitro de 8 antimicrobianos en 85 cepas de Shigella aisladas recientemente. Se observó una actividad moderada, aunque todavía adecuada de Ampicilina y Cotrimoxazol, los antimicrobjanos más usados en el tratamiento de la Shigellosis, con 75 y $78,8 \%$ de cepas sensibles. Cloramfenicol fue discretamente superior a los anteriores con niveles más bajos de CIM y $85,8 \%$ de cepas sensibles. Los aminoglicósidos demostraron actividad en todas las cepas, siendo superados solamente por las cefalosporinas de tercera generación ya que Cefotaxime $y$ Ceftriaxone inhibieron al total de cepas con CIM $+0,25 \mathrm{mcg} / \mathrm{ml}$.

La actividad de 8 antimicrobianos frente a 100 cepas de Salmonella typhimurium aisladas de coprocultivos de niños con diarrea, se indica en la Tabla 3. Amikacina y las cefalosporinas de tercera generación resultaron ser los antibióticos de mayor actividad con 100\% de cepas sensibles. Gentamicina y Dibekacina se ubicaron en una posición intermedia y Cotrimoxazol, Cloramfenicol y Ampicilina fueron los menos activos; en la proyección clínica estos 4 antibióticos resultaron utiles en la mitad (53-54\%) de las cepas estudia. das. 
Tabla 2 .

Actividad comparativa in vitro (CIM mcg/ml) de 85 cepas de Shigella sp frente a 8 antimicrobianos

$\begin{array}{llr}\text { CIM 50 } & \text { CIM 90 } & \text { \%cepas } \\ \text { mog/ml } & \mathrm{mcg} / \mathrm{ml} & \text { sensibles }\end{array}$

$\begin{array}{lrr}\text { Ampicilina } & 1 & \geqslant 256 \\ \text { Cotrimoxazole } & 2 & \geqslant 256 \\ \text { Cloramfenicol } & 2 & 64 \\ \text { Gentamicina } & 1 & 1 \\ \text { Dibekacina } & 1 & 2 \\ \text { Amikacina } & 2 & 4 \\ \text { Cefotaxine } & \$ 0.25 & \$ 0.25 \\ \text { Ceftriaxone } & \$ 0.25 & \$ 0.25\end{array}$

Tabla 3.

Actividad comparativa in vitro (CIM mcg/ml) de 100 cepas de Salmonella Typhinurium frente a 8 antimicrobianos

$$
\begin{array}{ll}
\text { CIM 50 } & \text { CIM } 90 \\
\mathrm{mcg} / \mathrm{ml} & \mathrm{mcg} / \mathrm{ml}
\end{array}
$$

\% cepas sensibles

Gentamicina
Dibekacina
Amikacina
Ampicilina
Cloramfenicol
Cotrimoxazole
Cefotaxime
Ceftriaxone

En la Tabla 4 se observa la actividad in vitro de 8 antimicrobianos en 83 cepas de Salmonella typhi aisladas de hemocultivos. Todas resultaron sensibles a $\operatorname{los} 8$ antibióticos probados, pero en términos de actividad, Cloramfenicol (que es la veces inferior al resto, y se observó alta efectivi. dad de Ampicilina y Cotrimoxazol.

\section{Tabla 4.}

Actividad comparativa in vitro (CIM $\mathrm{mcg} / \mathrm{ml}$ ) de 83 cepas de Salmonella typhi frente a 8 antimicrobianos

$\begin{array}{llr}\mathrm{CIM} \mathrm{50} & \mathrm{CNM} 90 & \text { \%cepas } \\ \mathrm{mcg} / \mathrm{ml} & \mathrm{mcg} / \mathrm{ml} & \text { sensibies }\end{array}$

$\begin{array}{lrrr}\text { Cloramfenicol } & 4 & 4 & 100 \\ \text { Cotrimoxazole } & \leqslant 0.25 & 1 & 100 \\ \text { Ampicilina } & \leqslant 0.25 & 1 & 100 \\ \text { Gentamicina } & \leqslant 0.25 & 0.5 & 100 \\ \text { Dibekacina } & 1 & 1 & 100 \\ \text { Amikacina } & 1 & 2 & 100 \\ \text { Cefotaxime } & \leqslant 0.25 & \leqslant 0.25 & 100\end{array}$

El comportamiento de las cepas resistentes se analiza en las Tablas 5, 6 y 7 . En ECEP $10,8 \%$ de droga de elección) resultó el menos activo, 4 a 20 las cepas mostraron resistencia a un solo antibiótico $(9,6 \%$ a Ampicilina y $1,2 \%$ a Cloramfenicol), las restantes mostraron resistencia múltiple, siendo los patrones más frecuentes la resistencia combinada a Ampicilina-Neomicina, seguida por el Ampicilina-Neomicina-Cotrimoxazol.

Tabla 5.

Patrón de resistencia de 83 cepas de ECEP

Resistentes A No cepas $\%$

Sólo Am

Sólo CAF

$\mathrm{Am}-\mathrm{CAF}$

Am - Neo.

Am - SXT

Am - Neo - SXT

Am - Neo - CAF

$A m$ - NeO - CAF - SXT

$A m-C A F-S X T-G m-D b$

TOTAL

Am = Ampicilina $; \mathrm{CAF}=$ Cloramfenicol;

Neo $=$ Neomicina.SXT

$\mathrm{SXT}=$ Cotrimoxazole $; \mathrm{Gm}=$ Gentamicina;

$\mathrm{Db}=$ Dibekacina.

Tabla 6.

Patrón de resistencia de 33 cepas de Shigella

Resistentes A

$$
\text { No cepas }
$$

Sólo Am

Sólo CAF

18.2

Sỏlo SXT

4

12.1

$A M$ - SXT

$\mathrm{Am}-\mathrm{CAF}$

$C A F-S X T$

Am - SXT - CAF

6.1
$A m-C A F-S K T$

Tabla 7.

Patrón de resistencia de 51 cepas de Saimonelia Thyphimurium

\begin{tabular}{lcr} 
Resistentes A & No cepas & $\%$ \\
Sólo Am & 3 & 5.9 \\
Sólo CAF & 2 & 3.9 \\
Am - Gm - Db & 1 & 2.0 \\
Am - Gm - Db - CAF & 3 & 5.9 \\
Am - Gm - Db - CAF - SXT & 42 & 82.3 \\
\hline TOTAL & 51 & 100.0
\end{tabular}


En las cepas Shigella (Tabla 6) se observó un variado patrón de resistencia, la mitad de ellas presentaban monoresistencia, siendo lo más frecuente resistencia a Cotrimoxazol, en la otra mitad de las cepas la conducta observada con mayor frecuencia fue resistencia doble a Ampicilina y Cotrimoxazol. Una propotción de $6,1 \%$ de las Shigellas estudiadas mostró resistencia múltiple a Ampicilina-Cotrimoxazol y Cloramfenicol, los 3 antibióticos que constituyen las alternativas terapéuticas habituales.

Las cepas de Salmonella typhimurium mostraron en su gran mayoría un patrón común đe resistencia múltiple a 5 antibióticos: Ampicilina-Gentamicina-Dibekacina-Cloramfenicol $y$ Cotrimoxazol (Tabla 7).

\section{COMENTARIOS}

Este estudio permitió evaluar la respuesta actual de las principales bacterias enteropatógenas a los antimicrobianos de uso común en el país, y puede ayudar a orientar el uso de antibióticos en las enfermedades que provocan.

Parece claro que Neomicina ha perdido actividad sobre las ECEP y esto puede explicarse por. que la mayoría de las cepas fueron aisladas en pacientes pediátricos del Area Metropolitana Oriente de Salud, donde en los últimos años se ha utilizado la Neomicina cono primera elección en las infecciones entéricas por el germen, lo que podría haber contribuido a seleccionar cepas resistentes. De acuerdo a los, resultados obtenidos, esta resistencia parece ser cromosómica pues tiene un perfil constante y la resistencia a Neomicina se asocia en forma variable con resistencia a diferentes antimicrobianos. Nosotros no observamos resistencia cruzada entre Neomicina $y$ otros aminoglicósidos.

Sobre estas observaciones recomendaríamos dejar de utilizar Neomicina en infecciones por ECEP en el Area Oriente durante un periodo de 2 a 3 años, reemplazándola por otro antibiótico de acción tópica como Polimixina (sulfato de colistin) con lo cual se espera que, al cabo de este tiempo, se podría recuperar Neomicina como opción terapéutica. Este cambio de conducta favorecería el manejo de las infecciones por ECEP, que constituyen la primera causa de diarrea aguda en niños menores de 2 años en nuestro pais, alcanzando una incidencia de $37,3 \%$ en verano y $19 \%$ en invierno ${ }^{3}$.

Las cepas de Shigella conservan sensibilidad adecuada a los antibióticos utilizados habitualmente en el tratamiento de ta Shigellosis -Ampicilina, Cotrimoxazol y Cloramfenicol- que tienen actividad sobre el 75 a $85 \%$ de las cepas.

Todos los demás antimicrobianos estudiados se mostraron activos en la totalidad de las Shigellas estudiadas.

Entre las cepas resistentes el comportamiento más frecuente fue resistencia a un solo antibióti$\infty$ y pocas cepas mostraron resistencia triple a Ampicilina-Cloramfenicol y Cotrimoxazol. En esta situación podría recomendarse el uso de aminoglicósidos por vía parenteral pero sólo en pacientes con diarrea grave y prolongada.

La mitad de las cepas de Salmonella typhimurium estudiadas resultaron resistentes a uno o más antíbióticos observándose que sólo Amikacina y las cefalosporinas de tercera generación (Cefotaxime y Ceftriaxone) son activas frente a la totalidad de las cepas estudiadas. En Salmone1la typhimurium predominó un patrón de multiresistencia en la mayoria de las cepas resistentes incluyendo Ampicilina-Gentamicina-Dibekacina - Cloramfenicol y Cotrimoxazol to que sugiere resistencia de origen plasmidial e identidad genética de ellas, con posibilidades de un reservorio común que podria ser intrahospitalario; para precisar este aspecto sería necesario un tipo de análisis que excede los objetivos de esta comunicación.

El comportamiento de Salmonella typhimu. rium hace necesario estudjos de sensibilidad in vitro a cada cepa cuando el germen está provocando infección sistémica, sólo así serja posible guiar la terapia antimicrobiana para que resulte eficaz.

Finalmente es tranquilizador observar que las cepas de Salmonella typhi siguen siendo muy sensibles a los antimicrobianos, pues no se detectó ninguna cepa resistente a las 8 drogas probadas, que mostraron excelentes indices de actividad; Cloramfenicol y Cotrimoxazol mantienen su posición como drogas de primera elección en el tratamiento de la fiebre tifoidea en nuestro medio.

Los resultados observados no son necesariamente extrapolables a bacterias enteropatógenas de las especies aquí incluidas que se aislen en otras áreas o regiones del país, ya que los factores condicionantes de resistencia pueden ser diferentes, siendo el más influyente el uso o abuso de antibióticos en las poblaciones, por la presión selectiva que ejercen sobre los gérmenes y por su posible acción como agentes inductores de resistencia. Por lo tanto es necesario que cada centro asistencial estudie periódicamente los patrones de resistencia de las cepas bacterianas aisladas en su ambiente de modo de mantener una vigilancia adecuada que evite la expansión de cepas multiresistentes en una comunidad.

\section{RESUMEN}

Se estudió la sensibilidad in vitro de 368 cepas 
de bacterias enteropatógenas aisladas de infecciones agudas, entre ellas se incluyeron 100 cepas de ECEP (E. coli enteropatógenos clásicos), 85 cepas de Shigella, 100 cepas de Salmonella typhimurium y 83 cepas de Salnonella typhi, frente a los antimicrobianos de uso habitual en nuestro país.

El estudio se efectuó determinando la concen. tración inhibitoria mínima (CIM) de Ampicilina, Cloramfenicol, Cotrimoxazol, Gentamicina, Dibekacina, Amikacina, Cefotaxime y Ceftriaxone; frente a las cepas de ECEP se incluyeron además Neomicina y Polimixina B. Se analizó el perfil de resistencia de cada cepa.

Los resultados mostraron que sólo el $46 \%$ de las cepas de ECEP mantienen su sensibilidad a Neomicina; Polimixina se mostró activa frente al $100 \%$. De las cepas de Shigella estudiadas entre 75 y $85,8 \%$ resultaron sensibles a los antimicrobianos utilizados en el tratamiento de la Shigellosis: Ampicilina, Cotrimoxazol y Cloramfenicol.

De las cepas de Salmonella typhymurium la mitad resultó resistente a uno o más antibióticos: Amikacina, Cefotaxime y Ceftriaxone fueron activos frente al $100 \%$. En las cepas resistentes predominó un patrón común de multiresistencia que incluia 5 antibióticos (Ampicilina, Gentamicina, Dibekacina, Cloramfenicol, Cotrimoxazol).

El $100 \%$ de las cepas de Salmonella typhi resultaron sensibles a todos los 8 antimicrobianos estudiados, Cloramfenicol y Cotrimoxazol mantienen buena actividad in vitro continuando vigentes como drogas de elección en el tratamiento de fiebre tifoídea.

\section{REFERENCIAS}

1 Mìnistetio de Salud de Chile. Depto. Programa de las
Personas. Seminario de Control de enfermedades diarreicas. Epidemiología y vigilancia de las enfermedades diarreicas 1980 .

2 Zacarías J., Spencer E., Prado V., Herskovic P. y col. Recuperación fecal de Rotavirus y otros enteropatógenos en lactantes menores de 1 año con diarrea aguda. Rev. Chil. Pediatr. 53: 111, 1982.

${ }^{3}$ Prado $V$, Broun $S$. Análisis de ECEP clásjco como causa endémica de diartea aguda en niños chilenos. Rev. Chil. Pediatr. 55: 171, 1984.

4 Ministerio de Salud de Chile. Depto. de Planificación Primario de Enfermedades de declaración obligatoria. Anuario 1980.

5 Prado V.. Mimica I.: Estudio comparativo doble ciego entre los efectos terapéuticos de Neomicina, Lactobacilo versus placebo en lactarites con infección enteral por E. coli enteropatógenos. Rev. Chil. Pediatr. 45: 143, 1974.

6 Prado V., Banfi A., Donoso E.: Evaluación clínico bacteriológica del uso de Cotrimoxazol en Shigellosis. Rev. Chil. Pediatr. 46: 25, 1975.

7 Prado V., Cohen J., Banfi A.: Amoxicilina en el tratamiento de niros con Shigellosis. Rev. Chil. Pediatr. 50: 27, 1979.

${ }^{8}$ Prado V., Coheis J., Harun A., Aguirre X., Díoz $M . C$. .: Estudio comparativo randomizado de la eficacia clínico bacteriológica de Mecillinan versus Cotrimoxazol en Shizellosis. Rev. Chil. Pediatr. 52: $118,1981$.

9 Alforo G.: Related R plasmids in Salmonella typhi strains isolated in Mexico city from clinical isolates. Rev. Lat. Amer. Microbiol. 19: 209, 1977.

10 Olarte J., Galindo E.: Salmonella typhi resistant to chloramphenicol, ampicillin and other antimicrobial agents: strains isolated during an extensive typhoid fever epidemic in Mexico. Antimicrob. Agents Chemother 4: 597, 1973.

11 Datla N., Olarte J.: R factors in strains of Salmonella typhi and Shigella dysenteriae 1 isolated during epidemics in Mexico: clasification by compatibility. Antimicrob Agents Chemother S: $310,1974$.

12 National Committee for clinical laboratory standards. L.S.A., 1982. 